\title{
El sismo del 19 de septiembre. ¿Cómo enfrentamos la crisis en Morelos, México?
}

\author{
The earthquake of September 19. \\ How have we faced the crisis in Morelos, Mexico?
}

Rafael Monroy Ortiz ${ }^{[]]}$ Rodrigo Flores Reséndiz ${ }^{[I]}$ Celia Minerva Jiménez Ortiz ${ }^{[\text {[II] }]}$ Rafael Monroy Martínez $z^{[\mathrm{V}]}$ Columba Monroy Ortiz ${ }^{[\mathrm{V}]}$ César Augusto González Bazán ${ }^{[\mathrm{VI}]}$

\section{Resumen}

Los sismos son poco predecibles; el 19 de septiembre de 2017, 32 años después de un evento igualmente grave, se presentó otro de magnitud 7.1. Los daños incluyeron una cantidad importante de víctimas y pérdidas materiales en la capital del país, así como en Morelos, donde fue el epicentro; el Estado tuvo efectos graves en 30 de 33 municipios. En este trabajo, se sistematizan las acciones de la sociedad post desastre en el contexto de la vulnerabilidad estructural, en términos de la pobreza, y la falta de prevención en la política pública. Para ello se describen las características de la afectación y la organización Universidad Autónoma del Estado de Morelos-Sociedad Civil ante la contingencia, evidenciando una forma no convencional de respuesta organizada.

Palabras clave: desastres por sismo; vulnerabilidad estructural; políticas de mitigación.

\begin{abstract}
Earthquakes are difficult to predict. On September 19, 2017, 32 years after an equally severe event, another one happened, of magnitude 7.1. Damages included an important death toll and material losses in the capital of Mexico, as well as in Morelos, where the epicenter was - the State had serious damages in 30 municipalities out of 33. In this article, we systematize society's actions after the disaster in the context of structural vulnerability, in terms of poverty and lack of prevention in the public policy. To achieve this, we describe the characteristics of the damages and the organization between the university Universidad Autónoma del Estado de Morelos and civil society to face the contingency, showing an unconventional form of organized response.
\end{abstract}

Keywords: earthquake damages; structural vulnerability; mitigation policies. 


\section{Introducción}

Algunas perspectivas vigentes en torno al estudio sobre el ambiente son el reconocimiento de su condición crítica, como consecuencia de la dinámica económica y social (IPCC, 2014) o los crecientes impactos en diversos sectores sociales (Unep, 2016). En ambos casos, es posible identificar dos formas interconectadas de crisis, incluyendo aquella donde se subraya el papel de la sociedad capitalista en el origen de dicha condición (Bellamy Foster y Clark, 2018), (Angus, 2016), así como el incremento de los costos derivados de ello (Vargas, 2002; Bello, 2017). En estricto sentido, este par crítico se refleja consistentemente como consecuencia de los procesos económico, es decir, es resultado de los patrones de producción y consumo que son ajustados para sostenerse temporal y regionalmente sin modificar sus criterios de aprovechamiento, siendo inherente a dicha racionalidad un entorno social y ambiental en permanente desequilibrio.

Las dificultades que el capitalismo impone a los factores de producción dada su intensidad de aprovechamiento, han Ilevado a instrumentar estrategias de adecuación, las cuales no implican necesariamente la transformación de las causas estructurales de dichos desequilibrios, por el contrario, están diseñadas para no afectar la racionalidad económica, y reduciendo los posibles costos de producción que estos generan. Esto refiere a una valoración económica de los efectos ambientales, la cual aplica con particular interés para eventos naturales extremos 0 desastres, dado que estos han ocasionado una serie de costos económicos derivados de la restauración o la reducción de riesgos, muchas de las veces afectando el funcionamiento económico mismo.

En este sentido, los eventos naturales extremos o desastres ${ }^{1}$ que no son de origen antropogénico implican para su análisis y valoración, la identificación de los riesgos a los que está sometida la población, pero principalmente sus bienes materiales o la actividad económica misma. En términos generales, los desastres naturales se incrementaron de 200 a 350 entre 1990 y 2016, mientras que su impacto directo en las muertes ocasionadas por estos pasó de poco más de 200 a 300 por cada millón de personas, lo cual representa un serio impacto a la sociedad, sin embargo, estos alcanzaron costos de restauración o recuperación por una cantidad de 150 billones de dólares (a precios de 2016); este valor demuestran una tendencia creciente para los siguientes años, según Guha-Sapir, Hoyois, Wallemacq y Below (2017).

El incremento de la mortalidad o la pérdida material ejemplifican el riesgo de los desastres naturales en la sociedad moderna; su tendencia genera un estatus de vulnerabilidad que requiere la instrumentación de estrategias de mitigación. Además de una implementación progresiva, ha prevalecido como propósito más importante, la reducción de sus crecientes costos aunque con ello, poco se aborden sus patrones de distribución o la falta de reconocimiento de los costos sociales y ambientales. Tal vez una discusión importante al respecto sea que la connotación de vulnerabilidad y riesgo implica también el reconocimiento de los sectores con menor capacidad de recuperación e incluso, los 
efectos de que son objeto cuando estos tienen una relación directa con el ambiente en el caso de campesinos e indígenas e incluso, sectores urbanos marginales.

En este sentido, la prevención de riesgos que son ocasionados por los desastres incluyen una serie estrategias, entre las que destacan, la generación de información, la divulgación, la participación de los sectores en los procesos de planeación e instrumentación de medidas de mitigación, así como la inversión en esquemas preventivos; por las circunstancias de los países subdesarrollados, para estos es relevante la reducción de la disparidad social, dado que en tales sectores prevalece la población más vulnerable (Global Facility for Disaster Reduction and Recovery, 2016). De hecho se reconoce que estas prácticas no han sido del todo efectivas en los gobiernos considerados débiles (United Nations Office for Disaster Risk Reduction, 2015), muchos de ellos localizados precisamente en el hemisferio sur.

Al mismo tiempo, los países subdesarrollados todavía registran problemas sociales estructurales que le significan un estatus de vulnerabilidad para desastres naturales. En el caso de latinoamérica por ejemplo, el número de personas en condición de pobreza se estima para el 2016 en 186 millones, lo cual representa $30.7 \%$ de la población total, mientras que la pobreza extrema afectó al 10\% de esta, es decir a 61 millones de personas (Cepal, 2017). La modificación de tales condiciones aun resulta una estrategia necesaria para muchas regiones (United Nations Development Programme, 2016); esto implica la prevención misma de riesgos o en su defecto, resarcir los impactos provocados por los desastres.
En términos generales, la debilidad de los gobiernos subdesarrollados o la vulnerabilidad estructural de la población reflejan el contexto en el que son instrumentadas las estrategias para prevenir los impactos derivados de los desastres naturales o en su defecto, para resarcir sus principales consecuencias. El caso de México, es útil para tal propósito en el marco de la escala de efectos ocurridos después del sismo del 19 de septiembre de 2017. La mayor parte del territorio mexicano es sísmico y de sus 4 categorías de riesgo, alrededor de $68.32 \%$ se localiza en un nivel alto de vulnerabilidad; al mismo tiempo, se estima que 55.3 millones de personas se encuentran en pobreza, de los cuales 31.5 es vulnerable por carencias sociales (Coneval, 2016). De esta forma, la perspectiva de la vulnerabilidad está construída con una parte del riesgo derivada de la localización, pero también con las condiciones sociales que hacen a la población susceptible de ser afectadas en desastres. Tan solo en términos de la frecuencia de los eventos es posible observar que en 2017 se registraron 26,123 , la mayor parte de ellos identificados entre 3 y 3.9 grados en la escala de Richter e incluso entre 2007 y 2017, se presentaron 52 sismos de una magnitud mayor a 6 grados, los cuales resultan la mayor amenaza para la población (SSS, 2017; Secretaría de Gobernación, 2018). Aunado a ello, la vulnerabilidad de origen estructural refleja las condiciones de riesgo en la sociedad, con implicaciones graves para su seguridad en el caso de los sismos; tan sólo en términos de la distribución territorial del riesgo se observa que 1.8 millones de hectáreas de suelo urbano están en las condiciones más desfavorables (Conabio, 2015), e incluso, $42.85 \%$ del total de personas en el rango de pobreza habitan en él (Coneval, 2016). 
En este contexto, es posible observar que dos de los factores propuestos para evaluar las condiciones de vulnerabilidad ante desastres ocasionados por sismos, tienen que ver la instrumentación de estrategias de prevención, incluyendo políticas, estándares, participación de los sectores sociales, inversión en la transformación de las condiciones de la población misma o por otro lado, las formas de abordar las circunstancias post desastre con el propósito de recuperar un relativo nivel de estabilidad (Global Facility for Disaster Reduction and Recovery, 2016).

El objetivo de este trabajo es sistematizar las acciones de la sociedad post desastre ocasionado por el sismo del 19 de septiembre de 2017 en Morelos, México, que es donde se localizó el epicentro y al mismo tiempo, fue uno de los estados más afectados a nivel nacional. Al respecto es necesario hacer dos precisiones; la primera es que las acciones de la sociedad son consideradas aquellas en las que participaron conjuntamente la Universidad Autónoma del Estado de Morelos (UAEM) y la sociedad civil organizada, quienes reaccionaron naturalmente ante la eventualidad y dieron seguimiento desde el primer día y 50 días posteriores a la crisis. Cabe destacar que durante el texto se hace referencia a la ausencia de ayuda provista por el Estado, aunque no se propone como objetivo revisar su trabajo preventivo puntualmente. La segunda es que derivado de la sistematización de dichas acciones, es posible obtener información directa de las condiciones de vulnerabilidad estructural de la sociedad, en términos precisamente de las carencias sociales propuestas por Coneval (2016).

Por otro lado y desde que cada caso de crisis por desastres refleja características particulares en términos regionales, según las condiciones de la población y el Estado, en la literatura es posible identificar diferentes ejemplos y niveles de intervención (Global Facility for Disaster Reduction and Recovery, 2017); para el ejemplo de Morelos, se sistematizan las acciones de la sociedad tras sismo, la cual es una entidad en la que no se había presentado un sismo de tales consecuencias, de forma que no existe antecedente alguno, pero al mismo tiempo. En consecuencia, aquello que ha sido denominado prácticas post sismo en realidad están confomadas por prácticas improvisadas por la sociedad civil con los respectivos rasgos particulares.

En este marco, la estrategia metodológica incluye las siguientes etapas: a) la caracterización del proceso de organización inicial en los primeros dos días, en términos de las actividades realizadas, incluyendo la formación de un centro de acopio de alcance regional en la UAEM, ${ }^{2}$ la cantidad de ayuda donada por la sociedad y la cantidad de vehiculos enviados con esta a las comunidades afectadas; b) la descripción de la asunción de la responsabilidad que implicó el establecimiento de un centro de operaciones para la determinación de la distribución de la ayuda a las comunidades diariamente, el cual se basó en la elaboración de un sistema de informacion geográfico, mismo que siguió un proceso por si mismo; y finalmente, c) la descripción del seguimiento durante los días subsecuentes según las categorías de ayuda por tipo y localidad.

Entre los principales hallazgos de este trabajo es que se dejó al descubierto aquellas carencias sociales descritas por 
Coneval, lo cual configura precisamente uno de los factores multidimensionales de la vulnerabilidad, como se discute en la literatura. En este razonamiento, la pobreza o algunos de sus más importantes rasgos que quedaron en evidencia fueron, el uso de materiales frágiles o perecederos en la vivienda, la localización en sitios de riesgo o sin el soporte urbano arquitectónico mínimo indispensable y por supuesto, una ausencia de estrategias provistas por el Estado en términos preventivos. Debido a ello, un sismo superior a 7 grados en la escala de Richter, como el sucedido el 19 de septiembre, descubrió la vulnerabilidad inherente a la pobreza estructural de los sitios en los que se observaron la mayor cantidad de daños, descritos en términos de la cantidad de vivienda derruida y la condición de pobreza de la poblacion que la habitaba. Respecto a la sistematización de las acciones tras sismo es posible comentar que estas se presentan en una versión suscinta de datos y lógica de actuación.

\section{Características del riesgo sísmico en México}

México se localiza en una región con alta actividad sísmica; al respecto, el Servicio Sismológico Nacional (2018), clasifica al territorio nacional en 4 zonas $(A, B, C, D)$ según su nivel de riesgo e intensidad de los eventos, sin embargo es posible observar que alrededor del $68.32 \%$ del territorio se considera alto, correspondientes a los tipos B, C, D, ver Mapa 1. Esto es ocasionado debido a la interacción de las placas Norteamerica, Cocos, Rivera, Pacífico y Caribe, las cuales son responsables de una cantidad importante de eventos anualmente; en este sentido, de 1990 a 2010 se calcula un promedio de 1,342 anualmente, el cual se ha visto incrementado en los últimos años. Tan sólo entre 2016 y 2017, se registraron en total 41,404 (Servicio Sismológico Nacional, 2018), lo cual significa una mayor frecuencia.

Para el caso mexicano, se observan dos condiciones importantes respecto a la vulnerabilidad ocasionada por eventos sísmicos; la primera tiene que ver con la intensidad y frecuencia de estos y la segunda, es precisamente la pobreza prevaleciente en la sociedad misma. En este sentido, entre 2007 y 2017 se registraron 52 sismos de una magnitud mayor a 6 grados, $^{3}$ la cual está en un límite donde el riesgo por colapso de estructuras urbano arquitectónicas se vuelve mayor; más aún, si estas corresponden a un nivel marginal y en una localización urbana poco consolidada. En este sentido, se estima que en el país existen 55.3 millones de personas en un estatus de pobreza, de los cuales 31.5 es vulnerable por carencias sociales (Coneval, 2016), lo cual representa precisamente un riesgo mayor para su seguridad ante este tipo de eventos.

\section{Vulnerabilidad estructural}

Los fenómenos naturales son eventos con capacidad para producir daños a la población, y cuya prevención es particularmente difícil en circunstancias de disparidad o pobreza como las que se describen para el caso mexicano. Desde 1998, se estimaba que $95 \%$ de las muertes relacionadas con desastres naturales 
Mapa 1 - Regiones sísmicas en México

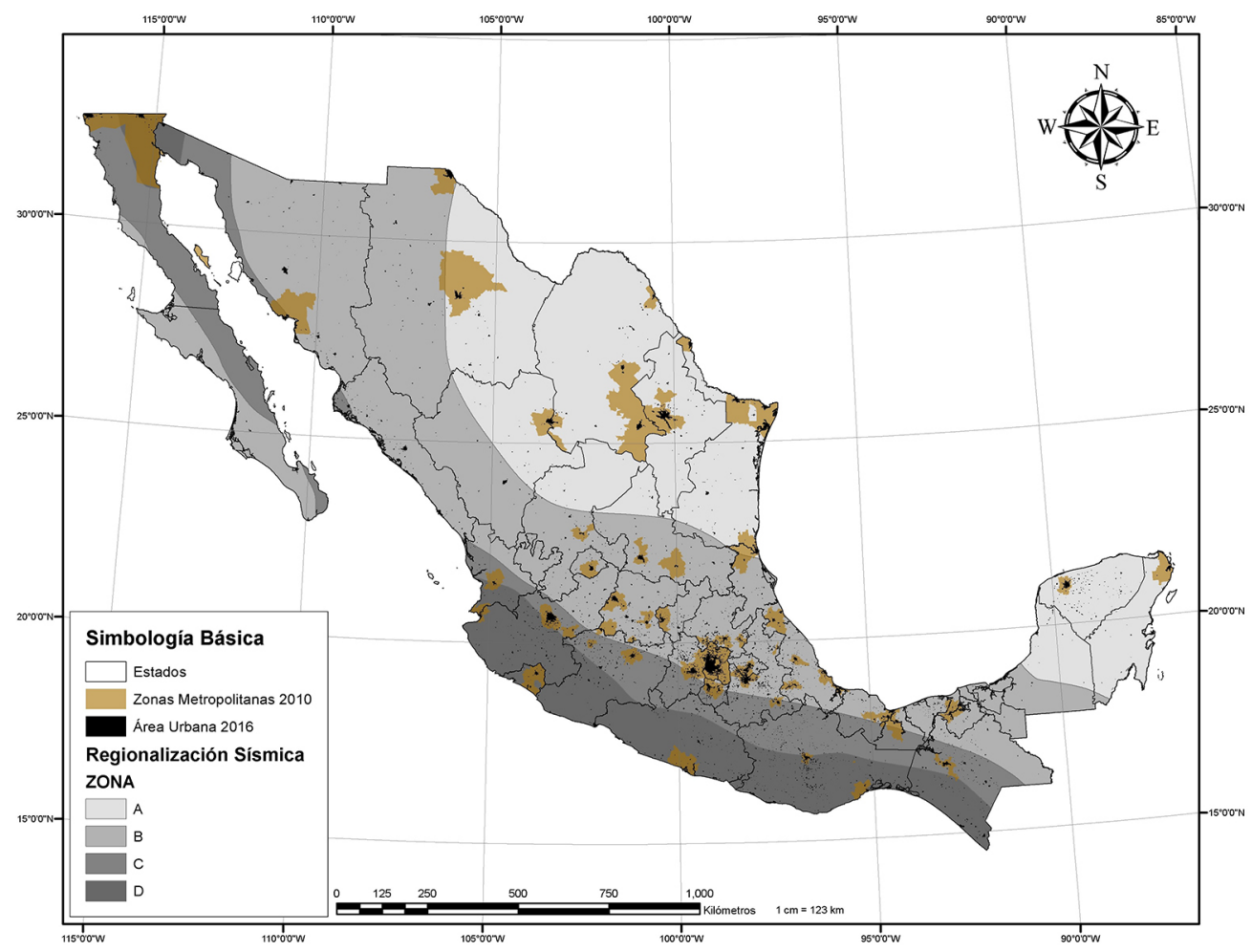

Fuente: elaboración propia basada en Servicio Sismológico Nacional (2018), Inegi (2018).

se concentraban principalmente en países subdesarrollados e incluso para 2000, ocasionó que alrededor de 19 millones de personas fueran desplazadas de sus hogares (BID-Cepal, 2000, p. 5).

Dados los problemas estructurales de la sociedad, es un hecho que los efectos de los desastres naturales son un problema en el hemisferio sur, y de manera directa en su sistema de asentamientos humanos poco consolidados. En el Mapa 2, se observan los epicentros mayores o iguales a 6 grados en la escala de Richter, según las condiciones de pobreza de la población estimadas por Coneval (2015); como se aprecia, el estatus de mayor pobreza es también donde existe mayor riesgo ocasionado por eventos sísmicos intensos. En septiembre se registraron dos sismos por arriba de 7 grados; el primero generó múltiples impactos en la región sur del país, particularmente Oaxaca, Guerrero y Chiapas, mientras que el segundo que se estudia en este trabajo, tuvo mayor afectaciones en la Ciudad de México y Morelos, lo cual vino a sumarse a la crisis en las entidades anteriormente mencionadas. 
Mapa 2 - Eventos sísmicos mayores a 6 grados en la escala de Richter y condición de pobreza

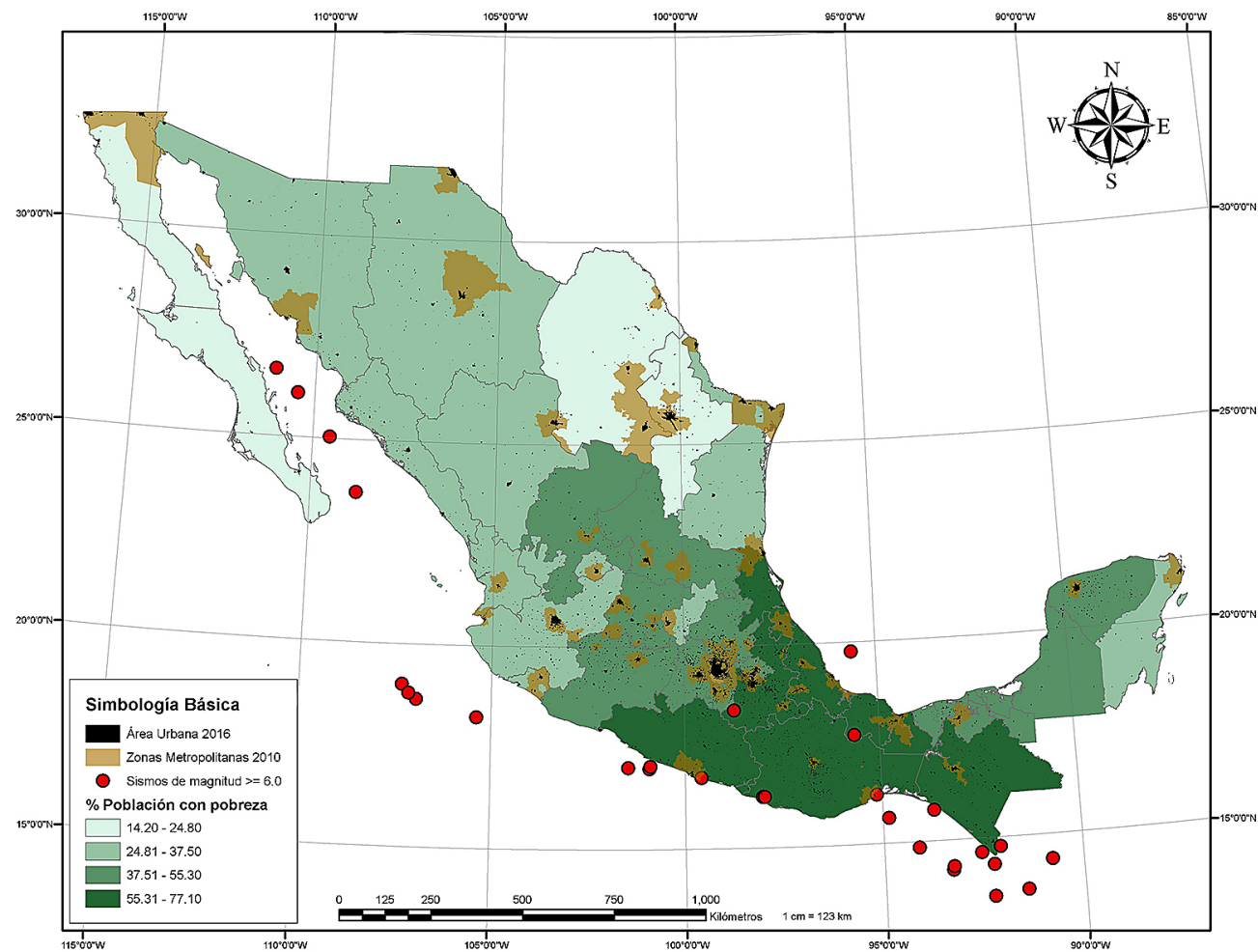

Fuente: elaboración propia basada en SSN (2018), Inegi (2018).

\section{Vulnerabilidad material}

Es de esperar que en las regiones con una alta concentración de pobreza, las condiciones de la vivienda reflejen suceptibilidad a eventos sísmicos, lo cual lleva a pérdidas materiales, que en el caso del 19 de septiembre fueron amplias. En consecuencia, los elementos de la vivienda son un factor determinante de dichos impactos, incluyendo, el tipo de material de construcción, el diseño, el tipo y peso de cubierta, la calidad constructiva y su estado de conservación (Izquierdo, 1999, p. 240); estos rasgos son identificados por Coneval como vulnerabilidad por carencias sociales.

Al mismo tiempo, la conservación de las construcciones depende de las condiciones sociales de la población que las habita; las construcciones deterioradas con el paso del tiempo y la falta de mantenimiento se encuentran en mayor riesgo. De hecho, estas pueden ser significativamente más débiles, lo suficiente como para incrementar su vulnerabilidad. Un ejemplo particular 
son las construcciones que tienen un daño previo a un evento natural, de manera que estos responden deficientemente a cualquier movimiento; en este caso, hasta una réplica débil puede causar daños desproporcionados, incluyendo colapso, entre edificios dañados por el sismo principal (CSE, 1998, p. 40). En otras palabras, el Diseño sismo resistente (DSR), que es una característica técnica para cumplir con el propósito de resistir una escala macrosísmica implica una revisión de las funciones de los distintos tipos de estructuras construidas, para lo cual no existe capacidad económica ni técnica en una proporción importante de la población.

\section{Centro de acopio. Universidad-sociedad civil}

El sismo del 19 de septiembre del 2017 dejó en claro que las políticas de prevención son necesarias para un país con tal nivel de actividad sísmica, y al mismo tiempo, que las experiencias previas no fueron suficientes para mejorar su instrumentación en momentos de crisis como el vivido. La primera reacción para el caso de Universidad Autónoma del Estado de Morelos (UAEM) fue la siguiente: el sismo dañó alrededor de 8 unidades académicas de la universidad, lo que llevó a suspender las clases por seguridad de la comunidad; la revisión estructural de los edificios llevó alrededor de 30 días, pero como parte del protócolo de protección las clases fueron suspendidas entre 40 y 50 días después del sismo.

La actividad académica de la UAEM colapsó desde el 19 de septiembre e incluso en términos laborales también, debido a un conflicto de meses entablado con el gobierno del estado, lo que dio como resultado que no se pagaran las quincenas correspondientes a los trabajadores durante los días más críticos del temblor; de hecho tuvieron que pasar 4 semanas antes de recibir el sueldo correspondiente, pero a pesar de ello, la UAEM y Sociedad Civil (SC) organizaron espontáneamente un centro de acopio en un auditorio dentro del campus con la capacidad suficiente para recibir víveres y todo tipo de ayuda.

\section{Organización inicial}

El 20 de septiembre, la población decidió voluntariamente darle la confianza a la Universidad y presentarse a colaborar en múltiples funciones, sin un orden establecido por protócolos, pero con una subdivisión del trabajo, según el tipo de responsabilidades; entre las más importantes destacan: a) el establecimiento de carpas con un mobiliario básico de mesas y sillas para recibir las donaciones; b) la recepción de donaciones de todo tipo, principalmente despensas, conformadas por alimentos, agua y medicamentos; c) la selección de las diferentes donaciones para su almacenaje; d) cargar hacia los sitios dispuestos para almacenar; e) cargar con víveres los vehículos de personas voluntarias; f) trasladar los víveres a las comunidades más afectadas de las que se tenía conocimiento; g) instalar un comedor comunitario para voluntarios que llegaban de las jornadas.

El primer día solo se sabía que se tenía que ayudar, ya que había municipios en la entidad que lo necesitaban urgentemente, 
Foto 1 - Centro de acopio (septiembre, 22, 2017)

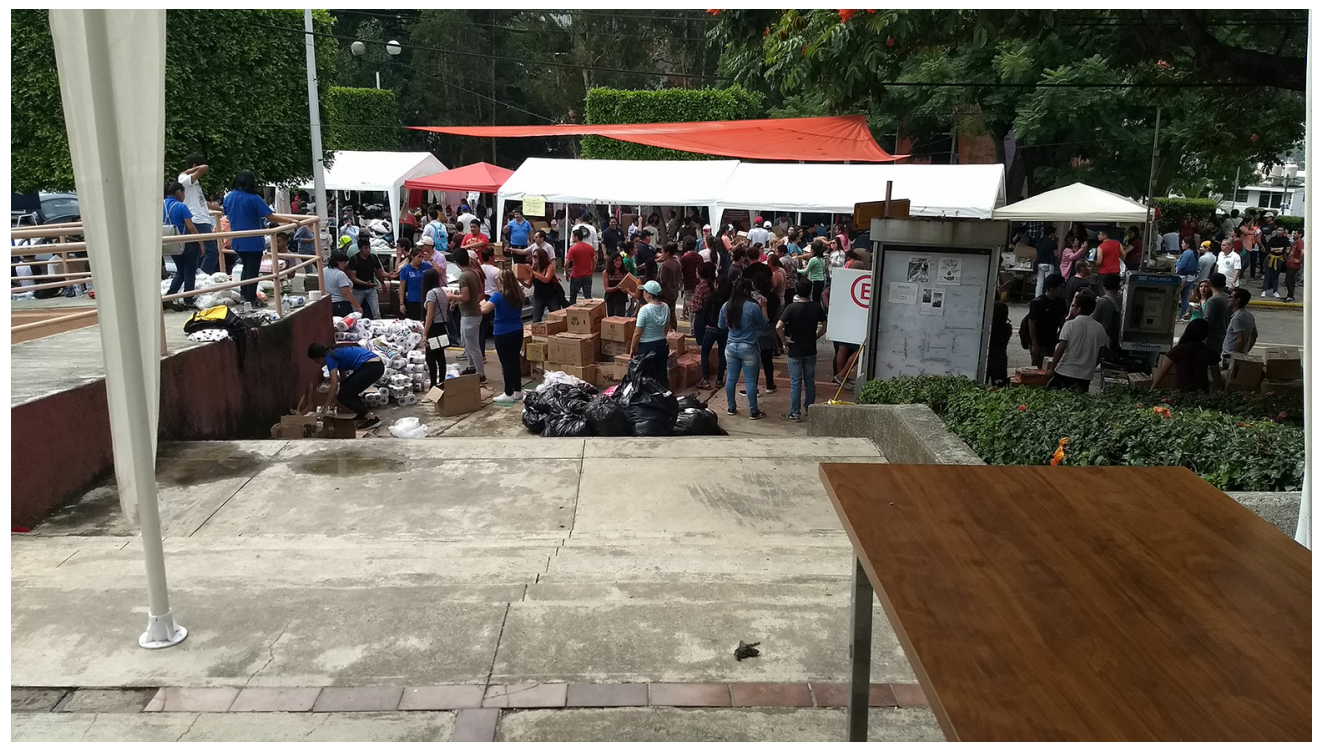

Fuente: toma propia.

Jojutla, Zacatepec, Axochiapán, Tetela del Volcán registraban pérdidas considerables. El día comenzó a las 7 de la mañana cuando comenzó a fluir la ayuda, terminaría a las 8 de la noche, al recibir a los últimos brigadistas.

El día 21 de septiembre se decide formar un sistema de información para el centro de acopio; las actividades del segundo día fluían, se recibían despensas y toda clase de ayuda, se llevaba a la bódega para clasificar y empacar. En su momento más difícil había 300 voluntarios clasificando, 100 recibiendo, 200 cargando vehículos e incluso había una fila de dos kilómetros de vehículos particulares y públicos de personas voluntarias para llevar víveres a los sitios más remotos y donde la crisis se presentaba gravemente.

El Colectivo de Estudios Urbano Ambientales de la Facultad de Arquitectura, que es una grupo organizado para la investigación científica dentro la unidad académica, se concentró a las afueras de los edificios para echar a andar dicho centro de información con la meta de identificar los sitios afectados y tratar de canalizar la ayuda con mayor claridad de las necesidades, ver Foto 2. El propósito final era dar una lógica a la ayuda de forma que los primeros resultados fueron mapeados con base en el uso de ARC GIS y publicados en formato 60, 90 centímetros frente el centro de acopio, para el conocimiento de la población, al mismo tiempo que se divulgó en las páginas oficiales de la universidad como puede corroborarse, sin embargo, no existía una lógica lo suficientemente definida aún.

Como puede apreciarse en el Mapa 3, existe un registro inicial de requerimientos contruido a partir de la información recabada por brigadistas que Ilevaron ayuda desde 
Foto 2 - Trabajo del centro de información

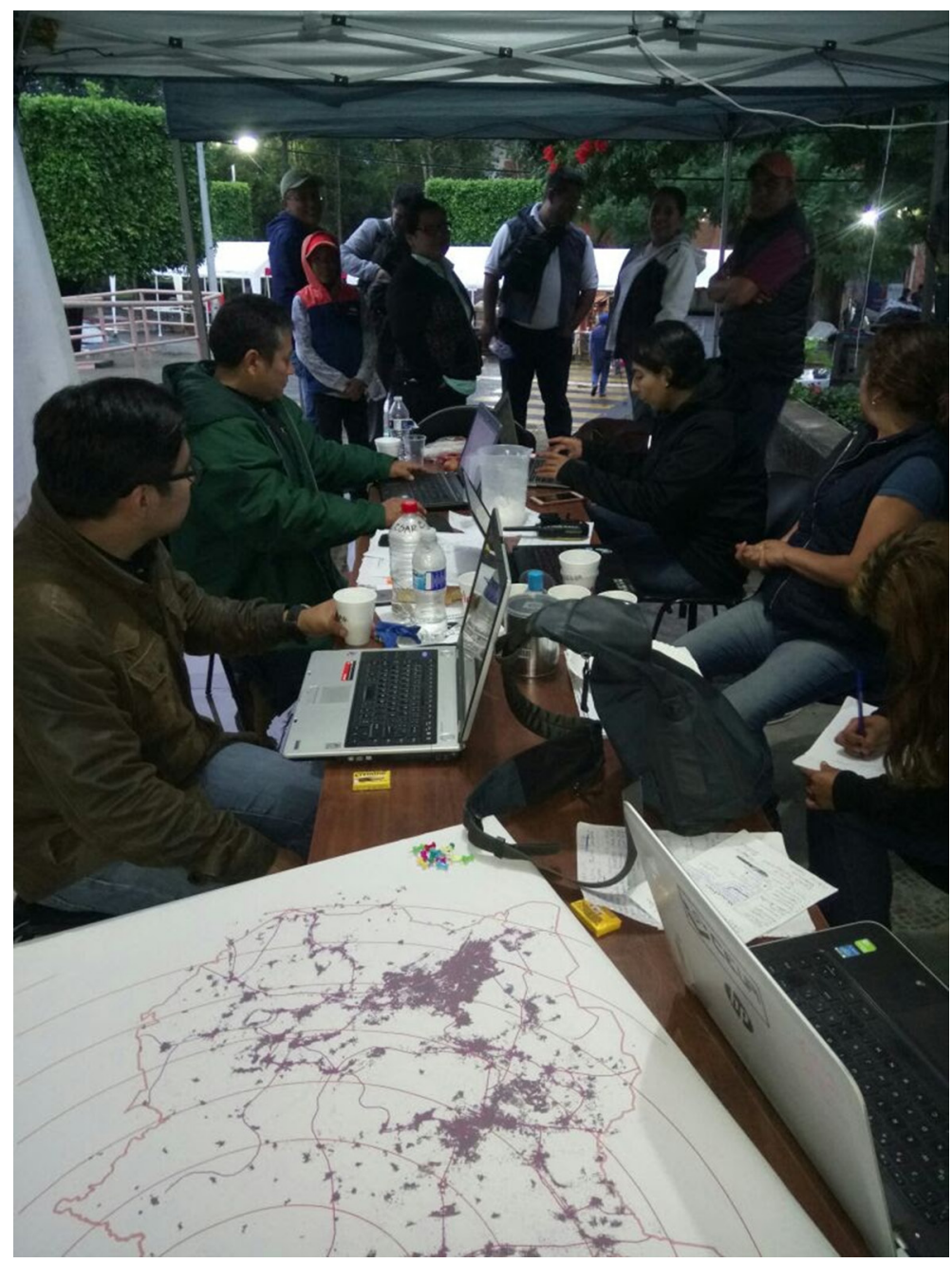

Fuente: toma propia.

el primer día y regresaban para comentar sus percepciones; esto dio la posibilidad de identificar los sitios de primera mano y con ello, plantear traslados organizados para la distribución de despensas. Sin embargo, la crisis se manifestaba por la necesidad de alimento, cobijo en zonas del norte de la entidad donde la temperatura mínima para el momento era debajo de $10^{\circ} \mathrm{C}$, particularmente cerca del volcán Popocatepetl, mientras que en el sur de la entidad con un registro arriba de $20^{\circ} \mathrm{C}$ se requerían medicinas en zonas que además habían perdido hospitales, es decir, existía una comprensión poco clara de necesidades que comenzaba a alimentarse con información de primera mano. 
Mapa 3 - Necesidad de ayuda por localidad (sitios identificados con efectos graves el 21 de septiembre)

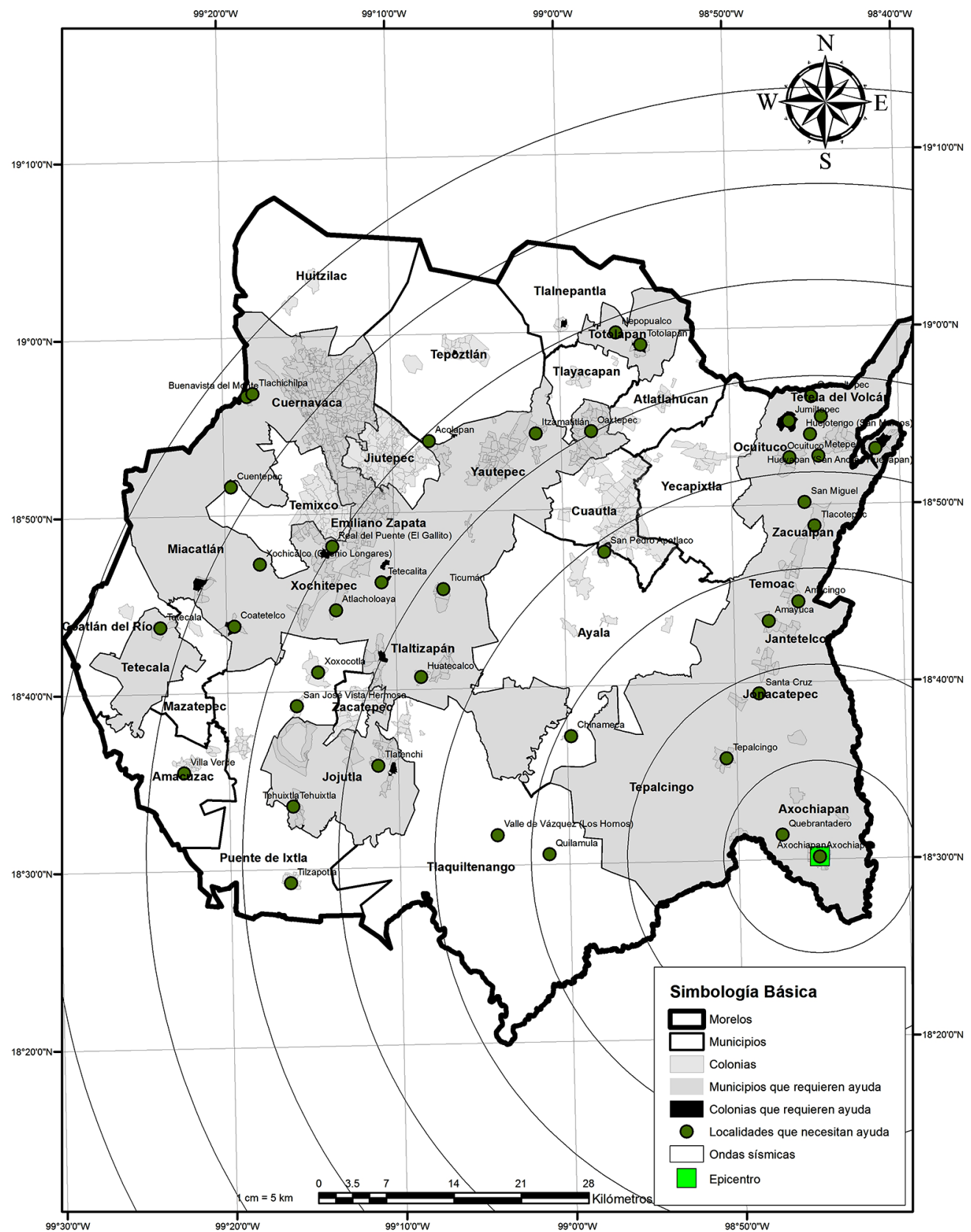

Fuente: elaboración propia basada en levantamiento propio; Inegi (2018). 


\section{La asunción de la responsabilidad y el seguimiento}

Como resultado de dos dinámicas concretas llevadas a cabo por la Universidad y la Sociedad Organizada, se logró lo siguiente: la población que voluntariamente llevaba ayuda a las poblaciones fue identificando mayor cantidad de sitios que también tenían requerimientos y a su regreso, lo informaban al centro de control, permitiendo con ello una mejor atención; al mismo tiempo, se constituyeron brigadas de académicos, estudiantes y población que ayudaban principalmente con la remoción de escombro en zonas que representaba un riesgo para la seguridad de las personas. Estos pequeños grupos eran trasladados con transporte universitario y con voluntarios de transporte público-privado que permitían llevar 30 o 40 personas por brígada. Al dia se trasladaron alrededor de 150 personas, ver Cuadro 1.

Tras todo trabajo conjunto, se tuvo la información necesaria para dar puntual seguimiento de la ayuda, ver Cuadro 2; en paralelo, el Estado desvíaba la ayuda para sus bódegas y después asumir el mismo la entrega a su nombre, secuestrando vehículos de estados del interior del país que envíaban toneladas. Al respecto, cabe destacar que las universidades del país, respondieron incluyendo Zacatecas, Aguascalientes, Coahuila, Colima, Nuevo León, Tamaulipas, San Luís Potosí, Chihuahua y otras tantas, que enviaron toda clase de ayuda e incluso, especialistas y voluntarios para trabajo en campo.

\section{Cuadro 1 - Ejemplo de registro de brigadistas ${ }^{4}$}

\begin{tabular}{|c|l|c|c|}
\hline $\begin{array}{c}\text { Brigadistas alumnos } \\
\text { 22/09/17 }\end{array}$ & \multicolumn{1}{|c|}{ Nombre } & Número teléfono & Facultad \\
\hline 1 & Mafer Gutiérrez & 77719913051 & Civil \\
2 & Cintia Ochoa & 7772991235 & Civil \\
3 & Daniela Ortega & 7772970268 & Civil \\
4 & Karina García & 7771839291 & Civil \\
5 & Monserat Acosta & 7772334336 & Civil \\
6 & Estef Palacios & 7772232069 & Civil \\
7 & Belem Gutiérrez & 7775646980 & Civil \\
8 & Itzel Alejandro & 7773052535 & Civil \\
9 & Mafer Sotelo & 7774922156 & Civil \\
10 & Karla Olea & 7441025579 & Civil \\
11 & Naomi Figueroa & 7775659350 & Civil \\
12 & Mariano Medina & 7772130611 & Civil \\
13 & Itzel Marroquin & 7774436086 & Civil \\
14 & Teresa de Jesús & 7773536565 & Civil \\
15 & Sandra Villegas & 7331355827 & Civil \\
16 & Elena Silva & 7774475276 & Civil \\
\hline
\end{tabular}

Fuente: registro hecho por la brigada de envío de voluntarios. 


\section{Cuadro 2 - Ejemplo de registro de ayuda por localidad Salida de material 26 de septiembre}

\begin{tabular}{|c|c|c|c|}
\hline Localidad & Responsable & Material & Cantidad \\
\hline Puente de Ixtla & Jazmín Rodríguez 7773609669 & $\begin{array}{l}\text { Despensas } \\
\text { Colchonetas } \\
\text { Medicamentos } \\
\text { Agua } \\
\text { Cascos } \\
\text { Guantes }\end{array}$ & $\begin{array}{l}5 p z \\
3 p z\end{array}$ \\
\hline Coatlán del río & Brigadas de apoyo jóvenes de facultad arquitectura & $\begin{array}{l}\text { Palas } \\
\text { Picos } \\
\text { Lonas } \\
\text { Cuerdas }\end{array}$ & $\begin{array}{l}15 p z \\
5 p z \\
5 p z \\
8 p z\end{array}$ \\
\hline Coatlán del río & Nidia 4615933730 fundación nutrición y vida & $\begin{array}{l}\text { Despensas } \\
\text { Ropa } \\
\text { Medicamentos }\end{array}$ & \\
\hline Coatlán del río & Brigadas de apoyo jóvenes de facultad psicología & Cascos & $4 \mathrm{pz}$ \\
\hline Tlayacapan & Brigadas de apoyo jóvenes de facultad arquitectura & Cascos & $23 \mathrm{pz}$ \\
\hline Campus Chamilpa & $\begin{array}{l}\text { Brigada de apoyo a peritaje alumno facultad de } \\
\text { arquitectura }\end{array}$ & Metro & $1 \mathrm{pz}$ \\
\hline Jojutla & Roger Mixcoac & $\begin{array}{l}\text { Despensas } \\
\text { Agua }\end{array}$ & \\
\hline Tlaquiltenango & Néstor Cabañas & Despensas & \\
\hline Tlaltizapan & Juventino Cabañas & Despensas & \\
\hline Tehuixtla & Gregorio Flores & Despensas & \\
\hline Amacuzac & $\begin{array}{l}\text { Manuel Horna empresa hp } 5539282368 \\
\text { camioneta acadia placas r19ags }\end{array}$ & $\begin{array}{l}\text { Despensas } \\
\text { Palas } \\
\text { Picos }\end{array}$ & $\begin{array}{l}2 p z \\
2 p z\end{array}$ \\
\hline Tepetzingo & Iván Flores facultad de agropecuarias 7771256435 & $\begin{array}{l}\text { Despensas } \\
\text { Palas } \\
\text { Picos }\end{array}$ & $\begin{array}{l}2 p z \\
2 p z\end{array}$ \\
\hline Coatetelco & Daniela Castillo 7773608777 & Despensas & \\
\hline Ocuilan Estado de México & Nelson ríos jefe de brigada forestal 7223716160 & Despensas & \\
\hline Acolapa & Víctor Castillo 5512937505 & Despensas & \\
\hline Ayala & Claudia Ortega 7352008177 & Despensas & \\
\hline
\end{tabular}

Fuente: registro hecho por la brigada de envío de ayuda.

Entre los mapas que se elaboraron se incluyeron una secuencia por tipo de ayuda y día según los requerimientos. Se estima que el traslado permitió cubrir al total de localidades afectadas incluyendo aquellas en las que era imposible acceder debido a los derrumbes o colapso de puentes y vías de comunicación. Es posible comentar que las necesidades más atendidas fueron víveres, agua, artículos de higiene personal y materiales para contruir refugios temporales, ver Mapa 4. 
Mapa 4 - Ejemplo de traslado de ayuda

en un día aleatorio de septiembre (agua)

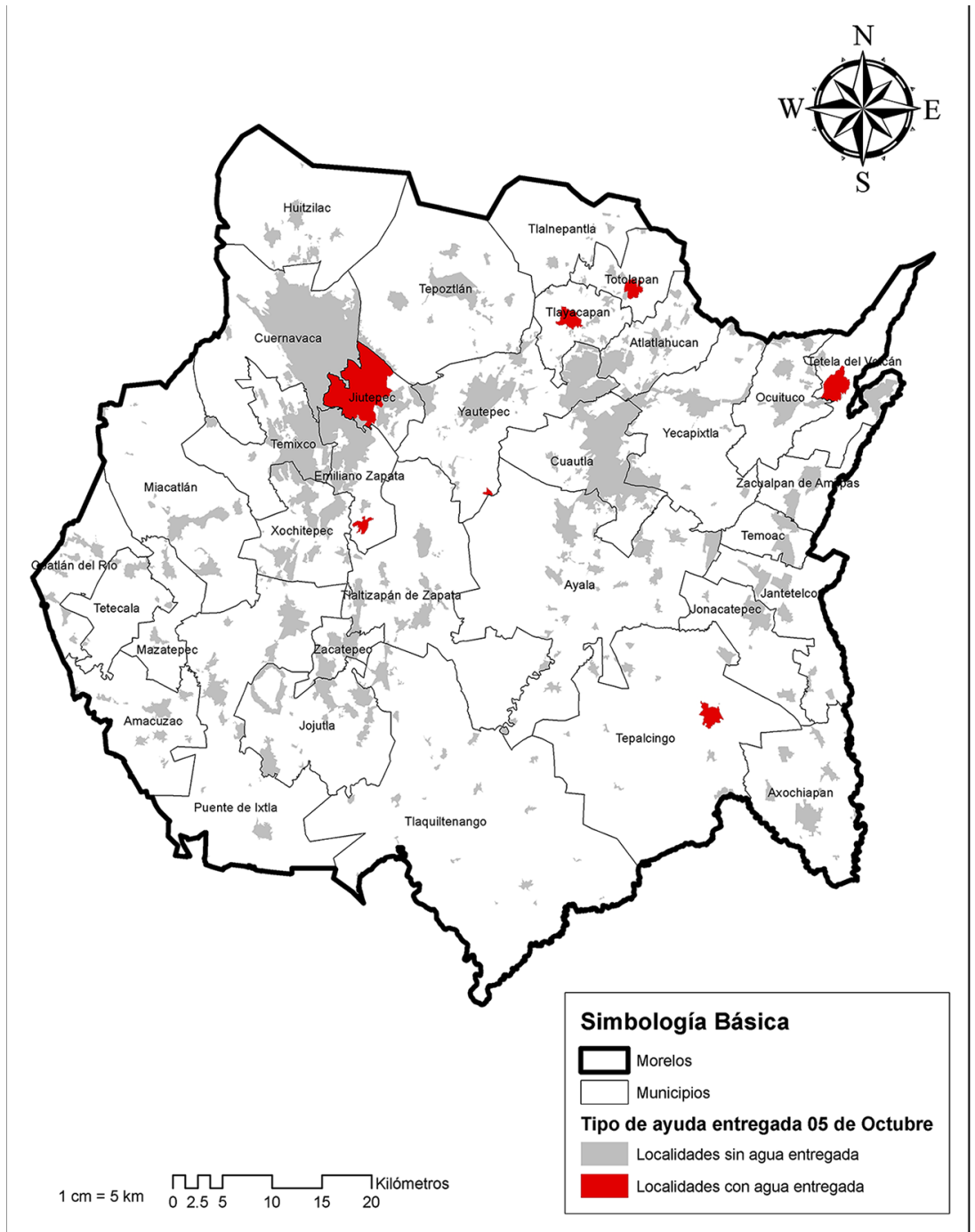

Fuente: elaboración propia.

\section{Los efectos, según condiciones de la sociedad}

El trabajo final del centro de acopio consistió en hacer un levantamiento de la cantidad de vivienda derruida por localidad y por municipio; para ello, hubo brigadistas que dejaron la función de traslado de víveres y se concentraron en fotografíar y georreferenciar la vivienda colapsada en al menos dos categorías, con pérdida total y con efectos graves, ver 
Mapa 5. Esto permitió hacer un plan de tercera etapa que no es descrito aquí, pero que tiene que ver con la atención multidimensional de los efectos, incluyendo aspectos médicos, psicológicos, arquitectónicos, urbanísticos y por supuesto de recuperación de la actividad económica, los cuales no han sido cubiertos en su totalidad, hasta la fecha.

En resumen, la hipótesis de que un factor de la vulnerabilidad es aquella situación estructural, particularmente de pobreza, es validado por el levantamiento de la vivienda derruida por el temblor para el caso de Morelos, ver Cuadro 3. Derivado de dicho levantamiento por localidad y munipio es posible observar que el grueso de la población que vio afectada su vivienda se encuentra en tal supuesto y la consecuencia del temblor se incrementó por la falta de políticas públicas de atención y seguimiento, como puede validarse también por las fallas de entrega de ayudas y apoyo con base en créditos para la reconstrucción, lo cual viene a agudizar los impactos ocasionados.

\section{Mapa 5 - Vivienda derruída en una localidad (Xoxocotla, Puente de Ixtla)}

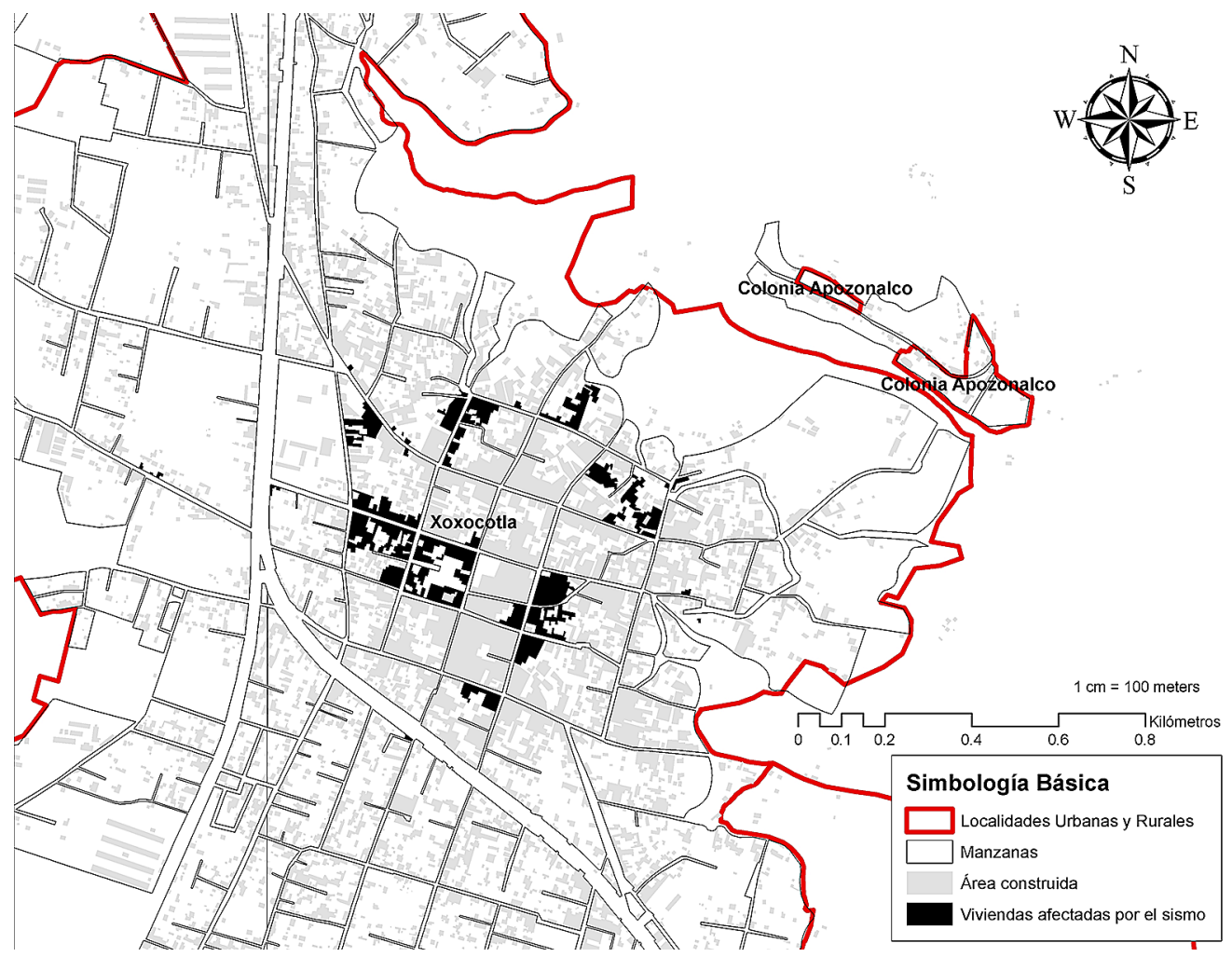

Fuente: elaboración propia basada en levantamiento de brigadistas. 
Cuadro 3 - Condiciones de pobreza, población y área edificada dañada

\begin{tabular}{|c|c|c|c|c|}
\hline Entidad & Municipio & \% Área dañada & Población total** & Grado de Marginación*** \\
\hline Morelos & Amacuzac* & 8.15 & 17,772 & Bajo \\
\hline Morelos & Atlatlahucan* & 9.49 & 22,079 & Medio \\
\hline Morelos & Axochiapan* & 13.84 & 35,689 & Alto \\
\hline Morelos & Ayala* & 7.16 & 85,521 & Bajo \\
\hline Morelos & Coatlán del Río & 15.19 & 9,768 & Bajo \\
\hline Morelos & Cuautla* & 12.55 & 194,786 & Bajo \\
\hline Morelos & Cuernavaca* & 7.16 & 366,321 & Muy bajo \\
\hline Morelos & Emiliano Zapata & 4.17 & 99,493 & Bajo \\
\hline Morelos & Huitzilac* & 7.16 & 19,231 & Bajo \\
\hline Morelos & Jantetelco* & 7.76 & 17,238 & Medio \\
\hline Morelos & Jiutepec* & 8.15 & 214,137 & Muy bajo \\
\hline Morelos & Jojutla* & 12.55 & 57,121 & Muy bajo \\
\hline Morelos & Jonacatepec & 7.76 & 15,690 & Bajo \\
\hline Morelos & Mazatepec* & 8.15 & 9,967 & Bajo \\
\hline Morelos & Miacatlán & 7.35 & 26,713 & Bajo \\
\hline Morelos & Ocuituco & 1.71 & 18,580 & Medio \\
\hline Morelos & Puente de Ixtla & 1.78 & 66,435 & Bajo \\
\hline Morelos & Temixco* & 8.15 & 116,143 & Bajo \\
\hline Morelos & Tepalcingo & 26.01 & 27,187 & Alto \\
\hline Morelos & Tepoztlán & 5.07 & 46,946 & Bajo \\
\hline Morelos & Tetecala* & 8.15 & 7,772 & Medio \\
\hline Morelos & Tetela del Volcán & 17.83 & 20,698 & Alto \\
\hline Morelos & Tlalnepantla & 12.05 & 7,166 & Alto \\
\hline Morelos & Tlaltizapán & 18.12 & 52,110 & Bajo \\
\hline Morelos & Tlaquiltenango & 7.16 & 33,844 & Bajo \\
\hline Morelos & Tlayacapan* & 9.49 & 17,714 & Bajo \\
\hline Morelos & Totolapan & 30.42 & 11,992 & Medio \\
\hline Morelos & Xochitepec & 1.6 & 68,984 & Bajo \\
\hline Morelos & Yautepec* & 9.49 & 102,690 & Bajo \\
\hline Morelos & Yecapixtla* & 12.55 & 52,651 & Medio \\
\hline Morelos & Zacatepec* & 12.55 & 36,159 & Bajo \\
\hline Morelos & Zacualpan* & 7.16 & 9,370 & Medio \\
\hline Morelos & Temoac* & 7.16 & 15,844 & Alto \\
\hline
\end{tabular}

* Municipios que se estimaron en base a los levantamientos en las zonas dañadas.

** Fuente: Inegi (2015).

***Fuente: Conapo (2015).

\section{Reflexiones preeliminares}

Las discusiones en torno al ambiente refieren particular atención a eventos naturales extremos o desastres que no son de origen humano; estos entrañan una dificultad de manejo condicionada por la posibilidad de disponer de información o de generar estrategias de prevención y tratamiento, aunque el caso de sismos representan serias dificultades técnicas. Es posible recuperar que las estrategias de manejo tienen entre sus ejes, la modificación de las condiciones de vida de la población, particularmente aquella 
identificada en el rango de pobreza; alcanzar esta meta es fundamental para prever las graves consecuencias que se presentan en dichos sectores.

En el caso de México, esta es considerada una región altamente sísmica; dichos eventos se presentan con una frecuencia e intensidad inusitada en los últimos dos años. De hecho, hasta 2010 el promedio no era mayor a 2000 anualmente, sin embargo entre 2016 y 2017 se registraron 25,000, lo que implica un constante riesgo y que ha puesto en evidencia, después del 19 de septiembre, precisamente la falta de una política pública al respecto.

Desde otra perspectiva, la etapa neoliberal del país ha ocasionado que la población registre un creciente deterioro de sus condiciones mínimas de vida, lo cual se ha visto reflejado en el incremento de la pobreza en términos absolutos, lo cual queda en evidencia en el informe de pobreza de Coneval. De hecho, el nivel de ingreso por debajo de las necesidades mínimas y considerado de los más bajos a nivel mundial y regionalmente, así como la constante pérdida de los beneficios sociales o de aquellos alcanzados por el estado de bienestar serían ejemplos pertinentes para el caso. Si bien, el mejoramiento de dichas condiciones es considerada una estrategia para mitigar los impactos de los desastres, para el caso mexicano, la sociedad se identifica más bien en un estatus de pobreza en poco más de la mitad de la población. La presencia de un evento de 7.1 en la escala de Richter significa exponer ambas condiciones, la falta de medidas preventivas, pero también las condiciones estructurales adversas de la población y el caso del 19 de septiembre resulta ejemplar para evidenciarlo.
Al mismo tiempo, la crisis no dejó opción a la espera de una ayuda institucional, la cual no sólo no llegó oportunamente a las zonas donde más se requería; de hecho, hasta el momento siguen pedientes. Por tanto, se obligó prácticamente a la población a reaccionar organizadamente; tal ejemplo queda evidenciado parcialmente en este trabajo, además de dejar claro que el potencial de resolución de la sociedad y la universidad pública funcionaron precisamente como sectores comprometidos con la crsis en ciernes. Incluso el estado asumió un papel de boicot para ayuda robando trailers que Ilevaban ayuda de otros estados, lo cual conmocionó al país entero por la mezquindad. De la misma foma, se busco impedir el levantamiento de vivienda afectada de diferentes formas, sin embargo la población logró avanzar en diferentes niveles.

Para los propósitos de este estudio quedo en evidencia que la población con mayores impactos, particularmente la pérdida de vivienda, fue aquella con mayores condiciones de pobreza, de manera que se registran 190 viviendas cada 1500 en los casos graves, excepto Jojutla y Zacatepec, donde los daños alcanzaron dimensiones de cuadras y colonias enteras. Sin embargo, con los registros de esta naturaleza provistos por brigadistas voluntarios, significa que las decisiones políticas no pasan por una comprensión de los problemas comunes en el sentido de solidarizarse por otros sectores sociales.

Finalmente, el caso del centro de acopio universidad-sociedad civil se sostuvo con el trabajo voluntario de cientos de personas al día, para funciones que incluyeron desde la 
recepción de despensas hasta la alimentación de los brigadistas que regresaban por la noche batidos en tierra. La forma en que se reaccionó durante la crisis fue común en la Ciudad de México, donde se tuvo que sacar a personas atrapadas después de días, hasta los ámbitos menos urbanos, pero con una afectación grave al funcionamiento de la vida cotidiana. En casos como el de Jojulta, una gran cantidad de damnificados sigue viviendo en tiendas de campaña, sin tener claridad del tiempo que falta para recuperar la normalidad.
La información de primera mano tanto de los registros como la sistematización de las etapas propuestas aquí del centro de acopio resulta de un valor insustituible para revisar protocolos de atención y estructuras de respuesta ante eventos naturales tan graves como el vivido por el sismo del 19 de septiembre y es interés de los autores compartirlo. Al mismo tiempo, no existe un registro para la entidad, de forma que puede sentar antecedentes para el planteamiento de medidas preventivas ante crisis.

[I] https://orcid.org/0000-0001-9970-1082

Universidad Autónoma del Estado de Morelos, Facultad de Arquitectura. Cuernavaca, Morelos, México. rafaelmoor@hotmail.com

[II] https://orcid.org/0000-0003-2069-9624

Universidad Autónoma del Estado de Morelos, Facultad de Arquitectura. Cuernavaca, Morelos, México. resendiz_barca@hotmail.com

[III] https://orcid.org/0000-0002-4653-7319

Universidad Autónoma del Estado de Morelos, Facultad de Arquitectura. Cuernavaca, Morelos, México. causa_270@live.com.mx

[IV] https://orcid.org/0000-0002-0813-8149

Universidad Autónoma del Estado de Morelos, Centro de Investigaciones Biológicas. Cuernavaca, Morelos, México.

ecologia@uaem.mx

[V] https://orcid.org/0000-0003-0066-992X

Universidad Autónoma del Estado de Morelos, Centro de Investigaciones Biológicas. Cuernavaca, Morelos, México.

columbam@hotmail.com

[VI] https://orcid.org/0000-0002-9157-4186

Universidad Autónoma del Estado de Morelos, Facultad de Arquitectura. Cuernavaca, Morelos, México. chin_gonzalez@hotmail.com 


\section{Notas}

(1) En definición, los desastres son considerados perturbaciones que pueden afectar variables ambientales, económicas y sociales en un territorio (Bello, 2017)

(2) La información generada en este trabajo fue elaborada por el Colectivo de Estudios Urbano Ambientales (CEUA) de la Facultad de Arquitectura de la UAEM, cuyas funciones fueron dar puntual seguimiento a la formación del centro de acopio y la distribución de la ayuda. El grueso de la información utilizada es resultado de dicho trabajo, es decir, proviene de informantes clave en el proceso, lo cual asegura su validez científica.

(3) Al momento de editar este documento, se presentó un sismo de 7.2 grados en la escala de Richter, por ejemplo. Características del reporte sísmico oficial. Magnitud: 7.2 Ocurrido el 2018-02-16 a las 17:39:38 horas (tiempo del Centro de México). Referencia de localización del epicentro: 11 $\mathrm{km}$ al sur de Pinotepa. Nacional, Oaxaca. Latitud: $16.25^{\circ}$. Longitud: $-98.03^{\circ}$. Profundidad: $12 \mathrm{~km}$. Réplicas: 827 (la mayor de M5.9) hasta las 14:00 horas del 2018-02-17. Recuperado de http:// www.ssn.unam.mx/.

(4) Todos los brigadistas fueron anotados con matrícula, teléfono e incluso ellos mismos, traían anotada su matrícula y tipo sanguíneo en el brazo izquierdo para prever eventualidades en su trabajo en campo. En el cuadro solo se anotan 17 de los 341 voluntarios del día.

\section{Referencias}

ANGUS, I. (2016). Facing the antropocene. Fossil capitalism and the crisis of the earth system. Nova York, Monthly Review Press.

BELLAMY FOSTER, J. y CLARK, B. (2018). The expropiation of nature. Monthly review, v. 10, n. 69, pp. 1-27.

BELLO, D. (2017). Desastres, crecimiento económico y respuesta fscal en los países de América Latina y el Caribe, 1972-2010. Revista de la CEPAL, n. 121, pp. 7-30.

BID - BANCO INTERAMERICANO DE DESARROLLO/CEPAL - COMISIÓN ECONÓMICA PARA AMÉRICA LATINA Y EL CARIBE (2000). Un tema del desarrollo: la reducción de la vulnerabilidad frente a los desastres. Santiago, BID-Cepal.

CEPAL - COMISIÓN ECONÓMICA PARA AMÉRICA LATINA Y EL CARIBE (2018). Panorama Social de América Latina, 2017. Santiago, Cepal.

COMISIÓN SISMOLÓGICA EUROPEA (CSE) (1998). Escala Macrosísmica Europea 1998. España, G. Grünthal.

CONAPO - CONSEJO NACIONAL DE POBLACIÓN (2018). Índice de marginación por entidad federativa y municipio 2015. Disponible en: https://www.gob.mx/conapo/documentos/indice-demarginacion-por-entidad-federativa-y-municipio-2015. Acceso en: 10 febrero 2018. 
CONEVAL - CONSEJO NACIONAL DE EVALUACIÓN DE LA POLÍTICA DE DESARROLLO SOCIAL (2013). Dimensiones de la seguridad alimentaria: evaluación estratégica de nutrición y abasto. México, Coneval.

(2015). Informe de pobreza en México, 2014. México, Coneval.

GLOBAL FACILITY FOR DISASTER REDUCTION AND RECOVERY (GFDRR) (2016). A decade of progress in disaster risk management. Washington, GFDRR.

(2017). Bringing resilience to scale. Washington, GFDRR.

GUHA-SAPIR, D.; HOYOIS, P.; WALLEMACQ, P. y BELOW, R. (2017). Annual Disaster Statistical Review 2016 The numbers and trends. Centre for Research on the Epidemiology of Disasters. Brussels, Centre for Research on the Epidemiology of Disasters (CRED) Institute of Health and Society (IRSS) Université catholique de Louvain.

INSTITUTO NACIONAL DE ESTADÍSTICA, GEOGRAFÍA E INFORMÁTICA - INEGI (2000). XII Censo general de población y vivienda. Aguascalientes, México, Inegi.

(2005). I/ Conteo de población y vivienda. Disponible en: http://www.beta.inegi.org.mx/ proyectos/ccpv/2005/default.html. Acceso en: 10 febrero 2018.

(2010). XIII Censo general de población y vivienda. Aguascalientes, México, Inegi.

(2018). Marco geoestadístico nacional. Disponible en: http://www.beta.inegi.org.mx/app/biblioteca/ ficha.html?upc=889463526636 [metadatos y mapoteca digital]. Acceso en: 12 febrero 2018.

INTERGOVERNMENTAL PANEL ON CLIMATE CHANGE - IPCC (2014). Climate change 2014: impacts, adaptation and vulnerability. Part A: Global and Sectoral aspects. contribution of working group II to the fifth assessment report of the intergovernmental panel on climate change. United Kingdom/New York, Cambridge University Press.

IZQUIERDO, A. (1999). Intensidad Macrosísmica. Física de la Tierra, n. 11, pp. 237-252.

SECRETARÍA DE DESARROLLO SOCIAL, CONSEJO NACIONAL DE POBLACIÓN, INSTITUTO NACIONAL DE ESTADÍSTICA, GEOGRAFÍA E INFORMÁTICA (2007). Delimitación de las zonas metropolitanas de México 2005. México, Sedesol/Inegi/Conapo.

SECRETARÍA DE GOBERNACIÓN - SEGOB (2017). Riesgos geológicos. Disponible en: https://www. sgm.gob.mx/Web/MuseoVirtual/Riesgos-geologicos/Sismologia-de-Mexico.html. Acceso en: 12 febrero 2018.

(2018). Sismología de México. Disponible en: https://www.sgm.gob.mx/Web/MuseoVirtual/ Riesgos-geologicos/Sismologia-de-Mexico.html. Acceso en: 12 febrero 2018.

SERVICIO SISMOLÓGICO NACIONAL - SSN (2018). Sismos. Disponible en: http://www2.ssn.unam. $\mathrm{mx}: 8080 /$ sismos-fuertes/. Acceso en: 12 febrero 2018.

UNITED NATIONS DEVELOPMENT PROGRAMME - UNDP (2016). Human Development Report 2016. Human Development for Everyone. Nova York, UNDP.

UNITED NATIONS ENVIRONMENT PROGRAMME - UNEP (2016). Global Gender and Environment Outlook. Nairobi, UN Environment. 
UNITED NATIONS OFFICE FOR DISASTER RISK REDUCTION - UNISDER (2015). Global Assessment report on disaster risk reduction. Making Development sustainable: the future of disaster risk management. USA, Unisder.

VARGAS, J. E. (2002). Políticas públicas para la reducción de la vulnerabilidad frente a desastres naturales y socionaturales. Santiago, Cepal.

Texto recebido em 19/fev/2018

Texto aprovado em 16/abr/2018 
